\title{
Management of future liver remnant: strategies to promote hepatic hypertrophy
}

\author{
Toru Beppu ${ }^{1,2}$, Kensuke Yamamuraa ${ }^{1,2}$, Hirohisa Okabe ${ }^{2}$, Tatsunori Miyata' ${ }^{2}$, Yuki Kitano ${ }^{2}$, Katsunori Imai ${ }^{2}$, \\ Hiromitsu Hayashi ${ }^{2}$, Shinichi Akahoshi ${ }^{1,2}$ \\ 1Department of Surgery, Yamaga City Medical Center, Yamaga 861-0593, Japan. \\ ${ }^{2}$ Department of Gastroenterological Surgery, Graduate School of Life Sciences, Kumamoto University, Kumamoto 860-8556, \\ Japan.
}

Correspondence to: Prof. Toru Beppu, Department of Surgery, Yamaga City Medical Center, 511, Yamaga 861-0593, Japan.

E-mail: tbeppu@yamaga-mc.jp

How to cite this article: Beppu T, Yamamura K, Okabe H, Miyata T, Kitano Y, Imai K, Hayashi H, Akahoshi S. Management of future liver remnant: strategies to promote hepatic hypertrophy. Hepatoma Res 2021;7:64. https://dx.doi.org/10.20517/23945079.2021 .49

Received: 10 Apr 2021 First Decision: 12 May 2021 Revised: 12 Jun 2021 Accepted: 29 Jun 2021 Published: 3 Sep 2021

Academic Editors: Allan Tsung, Ho-Seong Han Copy Editor: Yue-Yue Zhang Production Editor: Yue-Yue Zhang

\begin{abstract}
The resectability of hepatocellular carcinoma (HCC) has been assessed based on the liver functional test, the liver volume of the future liver remnant (FLR), and, more recently, the functional liver volume of FLR. Liver volume is estimated via multi-detector computed tomography and three-dimensional image visualization technologies, and functional liver volume is investigated via ${ }^{99 \mathrm{~m}} \mathrm{Tc}$-galactosyl human serum albumin scintigraphy, ${ }^{99 \mathrm{~m}} \mathrm{Tc}$-mebrofenin hepatobiliary scintigraphy, and gadoxetic acid-enhanced magnetic resonance imaging. Several special techniques have been developed to promote FLR hypertrophy, thus allowing for safe hepatectomy. As an interventional technique, portal vein embolization (PVE) is essential, and, along with transarterial chemoembolization or hepatic vein embolization, this is beneficial in promoting a much larger FLR. Dual embolization is recommended for patients with very small FLR or with PVE failure. Radioembolization by Yttrium-90 microspheres (i.e., radiation lobectomy) can help in achieving FLR hypertrophy and has an anticancer effect on HCC. Transarterial chemoembolization on PVE has a similar anticancer effect. Surgical procedures, such as two-stage hepatectomy as well as associated liver partition and portal vein ligation for staged hepatectomy, are somewhat invasive. Therefore, they should be applied as a salvage procedure for patients with $\mathrm{HCC}$ who had inadequate response to the interventional approach. However, the best approach should be selected mainly based on the functional volume of FLR and the patients' condition; in addition, the resources of each facility should be considered.
\end{abstract}


Keywords: Hepatectomy, future liver remnant, functional liver volume, portal vein embolization, transarterial chemoembolization, hepatic vein embolization, radiation lobectomy, two-stage hepatectomy, portal vein ligation for staged hepatectomy

\section{INTRODUCTION}

Liver resection is a widely adopted curative treatment for hepatocellular carcinoma (HCC ${ }^{[1,2]}$. The liver resectability of HCC is usually determined based on the preoperative liver function and future liver remnant (FLR) volume $\mathrm{e}^{[3-6]}$. The former is assessed by indocyanine green (ICG) tolerance test, while the latter is assessed using computed tomography $(\mathrm{CT})$ volumetry ${ }^{[3-7]}$. A clinically significant portal hypertension (hepatic venous pressure gradient $\geq 10 \mathrm{mmHg}$ ) had been regarded as contraindication to liver resection ${ }^{[1]}$. However, recent data show that liver resection is also safe in patients with significant hypertension depending on the model for end-stage liver disease score and the extent of liver resection ${ }^{[s, 9]}$. In addition, evaluation of liver stiffness by transient elastography has been shown to have a good specificity and sensitivity for prediction of postoperative decompensation ${ }^{[10]}$. Limited resection is recommended for patients with HCC in damaged liver; however, major hepatectomy is required for HCC patients with large tumor, as well as accompanying ipsilateral satellite nodules and vessel invasion.

Nowadays, functional volumetry can be assessed using ${ }^{99 m}$ Tc-galactosyl human serum albumin (GSA) scintigraphy, single-photon emission computed tomography (SPECT), ${ }^{99 \mathrm{~m}} \mathrm{Tc}$-mebrofenin hepatobiliary scintigraphy, and gadoxetic acid (Gd-EOB-DTPA) enhanced magnetic resonance imaging ${ }^{[1-15]}$. Segmental liver function can then be investigated using these images.

To promote FLR hypertrophy, several interventional and surgical approaches have been developed. Portal vein embolization (PVE) is one method used to obtain a larger FLR volume to expand the safety zone of hepatic resection without increasing morbidity and mortality ${ }^{[16-22]}$. In patients with HCC, PVE has other oncological advantages such as the prevention of intraportal metastases, the avoidance of extension of portal vein tumor thrombosis to the non-embolized area, and the augmentation of the antitumor effect of transarterial chemoembolization $(\mathrm{TACE})^{[23-25]}$. Moreover, additional TACE and hepatic vein embolization (HVE) during PVE can promote a greater increase in FLR in comparison with PVE alone ${ }^{[26-35]}$. On the other hand, radiation lobectomy (RL) with internal radiation therapy by Yttrium-90 microspheres is a novel treatment that can achieve hypertrophy of FLR as well as be therapeutic for HCCs in the embolized $\operatorname{liver}^{[36-38]}$. For patients with bilateral unresectable HCCs, surgical approaches such as one-stage hepatectomy following PVE, two-stage hepatectomy (TSH), and associated liver partition and portal vein ligation (PVL) for staged hepatectomy (ALPPS) are performed ${ }^{[39-43]}$.

This review summarizes the therapeutic methods that promote hepatic hypertrophy of FLR with the goal of safely meeting the indication for hepatectomy.

\section{ASSESSMENT OF FUTURE LIVER REMNANT}

FLR is usually assessed via CT volumetry ${ }^{[3-6]}$. Nowadays, preoperative simulations of the resected liver area volume can be correctly estimated with the use of three-dimensional image visualization technologies ${ }^{[44-46]}$, such as the SYNAPSE VINCENT medical imaging system (Fujifilm Medical, Tokyo, Japan), a popular method for three-dimensional visualization and virtual liver resection. 
In East Asia, especially in Japan, functional volumetry using ${ }^{99 m}$ Tc-GSA scintigraphy SPECT has been developed to assess partial liver function in patients undergoing PVE, followed by liver resection ${ }^{[47-50]}$. The liver is the only uptake site of ${ }^{99 \mathrm{~m}} \mathrm{Tc}$-GSA, making it a suitable agent for the assessment of liver function. Furthermore, the uptake of ${ }^{99 \mathrm{~m}} \mathrm{Tc}$-GSA is not affected by high levels of serum bilirubin. We recently established a novel combined ${ }^{99 m}$ Tc-GSA scintigraphy SPECT/CT fusion system that can more accurately determine functional liver volume ${ }^{[51]}$ [Figure 1].

Perioperative changes in functional liver volume are also estimated via ${ }^{99 m}$ Tc-hepatobiliary scintigraphy ${ }^{[52-54]}$. The hepatic uptake of mebrofenin can be calculated similar to ICG, and, thus, the mebrofenin uptake rate strongly relates with the ICG clearance test. The combination of dynamic hepatobiliary scintigraphy and SPECT-CT can provide accurate quantitative information regarding segmental FLR function ${ }^{[54]}$. Hepatobiliary scintigraphy is widely used mainly in Western countries, but it is not usable for patients with hyperbilirubinemia. Furthermore, preoperative measurements of ${ }^{99 \mathrm{~m}} \mathrm{Tc}-\mathrm{GSA}$ and ${ }^{99 \mathrm{~m}} \mathrm{Tc}$-mebrofenin uptake in the FLR are both more accurate in predicting postoperative liver failure and liver-related mortality compared to preoperative FLR volumetry by $\mathrm{CT}^{[52-55]}$.

Gd-EOB-DTPA-enhanced MRI allows for the assessment of both liver anatomy and function ${ }^{[14,15]}$. Meanwhile, quantitative multiparametric MRI can help in assessing individualized patient risk as part of the clinical decision-making process for liver cancer surgery ${ }^{[56]}$. However, the use of Gd-EOB-DTPA-enhanced MRI for the assessment of segmental liver function is still under debate. There are still some technical difficulties regarding the molar quantity of tracer required for visualization on MRI, as well as issues concerning the definite quantification of function ${ }^{[54]}$. If these problems were resolved, Gd-EOB-DTPAenhanced MRI might become the first choice for imaging because the MRI machine is more widely available than the two types of scintigram machine.

\section{STRATEGIES TO PROMOTE HEPATIC HYPERTROPHY}

PVE can be considered first-line therapy ${ }^{[20-24]}$, and additional TACE or HVE is recommended for patients with insufficient FLR after PVE alone or for those with very small initial FLR ${ }^{[26-35]}$. PVE + TACE and RL had definite advantages when treating HCC in the embolized liver ${ }^{[27-30,36-38]}$. Surgical procedures should be strictly applied based on the FLR functional reserve as a salvage option in selected patients with HCC in whom the interventional approach has been unsuccessful ${ }^{[42]}$.

\section{PORTAL VEIN EMBOLIZATION}

PVE for HCC was first introduced in 1986 by the Osaka City University group in Japan ${ }^{[16]}$. It was developed mainly to achieve hypertrophy of the FLR together with the atrophy of the embolized liver. The liver parenchyma is nourished by a dual blood supply of the hepatic artery and portal vein and has arterioportal communications, allowing PVE to be safely performed without liver infarction ${ }^{[57]}$. Patients with large HCCs sometimes have portal tumor thrombus, so they usually do not need PVE. However, patients with HCC demanding PVE can exist when extensive resection is required in comparison with their liver functional reserve $^{[22-25]}$.

The summarized volume data of PVE in Table 1 are limited to papers including only patients with HCC. The median percent of nontumorous remnant liver volume (\%LV) before PVE was 37\% (23\%-39.5\%), the median increment of \%LV was $11 \%$ (10.2\%-17.2\%), and the median interval between pre- and post-PVE evaluation was 24.5 (21-45) days. Differences in embolic materials, embolization procedures, or assessment time may have influenced these results. Incomplete obstruction or recanalization rates were relatively low in patients who underwent PVE with ethanolamine oleate or absolute ethanol ${ }^{[58-60]}$. These embolic materials 
Table 1. Changes in liver volume for patients with hepatocellular carcinoma undergoing portal vein embolization

\begin{tabular}{|c|c|c|c|c|c|c|}
\hline & Ref. & Publish year & No. of patients & Pre-PVE \%LV & Increment of \%LV & Interval of PVE to CT evaluation (days) \\
\hline 1 & Tanaka $^{[23]}$ & 2000 & 33 & 37 & 12 & 21 \\
\hline 2 & Palavecino ${ }^{[20]}$ & 2009 & 21 & 23 & 10.8 & NA \\
\hline 3 & Okabe $e^{[24]}$ & 2011 & 19 & 37.8 & 17.2 & 28 \\
\hline 4 & Siriwardana ${ }^{[21]}$ & 2012 & 34 & 23 & 11 & 45 \\
\hline 5 & $\mathrm{Beppu}^{[22]}$ & 2016 & 148 & 39.5 & 10.2 & 21 \\
\hline \multicolumn{3}{|c|}{ Median (range) } & $33(19-148)$ & $37(23-39.5)$ & $11(10.2-17.2)$ & $24.5(21-45)$ \\
\hline
\end{tabular}

LV: Non-tumorous remnant liver volume; PVE: portal vein embolization; NA: not available.

\section{Pre-PVE}

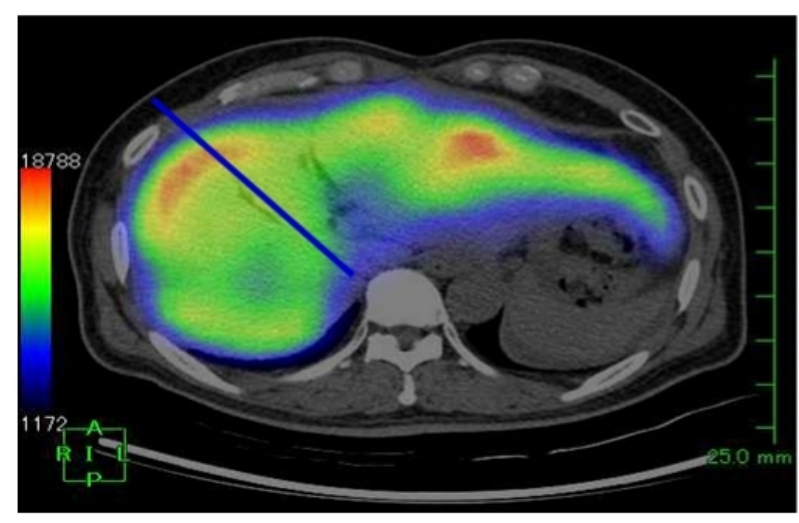

Post-PVE

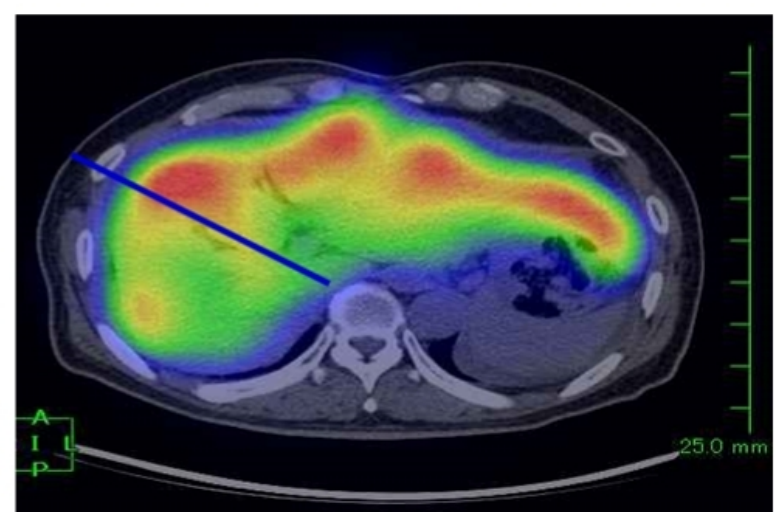

Figure 1. Fusion images of the ${ }^{99 \mathrm{~m}} \mathrm{Tc}-\mathrm{GSA}$ scintigraphy SPECT/CT before and after portal vein embolization (PVE). The non-embolized liver (left) became hypertrophic and hyperfunctional after PVE (right). The blue line is the cutting line of right hepatectomy.

can permit rapid and absolute damage of the portal vein vascular endothelium.

Liver regeneration was accomplished in a two-step manner after PVE, followed by major hepatectomy ${ }^{[61,62]}$. Liver regeneration and atrophy can also be assessed by both CT volumetry and functional volumetry with ${ }^{99 m}$ Tc-GSA scintigraphy SPECT/CT fusion system ${ }^{[51]}$. PVE may be able to not only achieve a larger FLR volume but also preserve a larger functional volume of the $\mathrm{FLR}^{[47-51]}$. Our study ${ }^{[51]}$ clearly demonstrated that the increment of the \%LV was $13.9 \%$ and the increment of functional liver volume (\%FLV) was $21.4 \%$ in patients undergoing right PVE. Before PVE, the correlation between the \%LV and the \%FLV was almost equivalent with a regression coefficient of $1.005(P<0.0001)$. By contrast, after right PVE, there was a strong significant correlation between \%LV and \%FLV, but the regression coefficient was $1.192(P<0.0001)$. The \%FLV is estimated to be about $20 \%$ larger than the \%LV after successful right PVE. Thus, the true liver function post-PVE can be possibly underestimated by traditional CT volumetry.

\section{ADDITIONAL TRANSARTERIAL CHEMOEMBOLIZATION ALONGSIDE PORTAL VEIN EMBOLIZATION}

For patients with HCC, TACE was additionally performed alongside PVE to increase FLR volume and/or to avoid tumor progression during the waiting period for major hepatectom ${ }^{[26-31]}$. There are currently no randomized controlled trials in this field. The pre-PVE \%LV were comparable across both groups [Table 2]. On the other hand, the increments of \%LV were significantly larger in the PVE + TACE group compared with PVE alone. The resectability rate was relatively high in the PVE + TACE group compared with PVE 
Table 2. Liver hypertrophy after hepatectomy for hepatocellular carcinoma patients undergoing PVE + TACE vs. PVE alone

\begin{tabular}{|c|c|c|c|c|c|c|c|c|c|}
\hline & Ref. & $\begin{array}{l}\text { No. of } \\
\text { patients }\end{array}$ & $\begin{array}{l}\text { Preoperative } \\
\text { therapy }\end{array}$ & $\begin{array}{l}\text { Pre-PVE } \\
\% \text { LV } \\
\text { (range) }\end{array}$ & $\begin{array}{l}\text { Increment } \\
\text { of } \% \mathrm{LV}\end{array}$ & $\begin{array}{l}\text { Comparison } \\
\text { of data }\end{array}$ & $\begin{array}{l}\text { Resection } \\
\text { rate }(\%)\end{array}$ & $\begin{array}{l}\text { Comparison } \\
\text { of data }\end{array}$ & $\begin{array}{l}\text { Interval of } \\
\text { PVE and HR } \\
\text { (days) }\end{array}$ \\
\hline \multirow[t]{2}{*}{1} & Ogata $^{[27]}, 2006$ & 18 & $P V E+T A E$ & $\begin{array}{l}30 \pm 6(21- \\
43)\end{array}$ & $12 \pm 5$ & $P=0.013$ & NA & NA & 37 \\
\hline & & 18 & PVE alone & $\begin{array}{l}29 \pm 5(23- \\
40)\end{array}$ & $8 \pm 4$ & & NA & & 40 \\
\hline \multirow[t]{2}{*}{2} & $Y_{o o}^{[28]}, 2011$ & 71 & $\mathrm{PVE}+\mathrm{TAE}$ & $41.4 \pm 7.3$ & $7.3 \pm 3.6$ & $P=0.035$ & 96 & $P=0.307$ & 29 \\
\hline & & 64 & PVE alone & $40.3 \pm 8.1$ & $5.8 \pm 4.5$ & & 91 & & 31 \\
\hline \multirow[t]{2}{*}{3} & $\begin{array}{l}\text { Terasawa }^{[29]} \\
2020\end{array}$ & 23 & $P V E+T A E$ & $\begin{array}{l}529(240- \\
1450)^{\#}\end{array}$ & $43(11-138)^{\star}$ & $P=0.035$ & 91 & $P=0.08$ & 48 \\
\hline & & 28 & PVE alone & $\begin{array}{l}568(253- \\
1227)^{\#}\end{array}$ & $31(4-72)^{\star}$ & & 68 & & 47 \\
\hline \multirow[t]{2}{*}{4} & $\operatorname{Park}^{[30]}, 2020$ & 109 & $\mathrm{PVE}+\mathrm{TAE}$ & 34 & $17.5 \pm 6.6$ & $P=0.001$ & NA & NA & 39 \\
\hline & & 38 & PVE alone & 34 & $12.1 \pm 5.9$ & & NA & & 23 \\
\hline
\end{tabular}

${ }^{\#}$ Future remnant liver volume $(\mathrm{mL})$. ${ }^{\star}$ The percentage of future remnant liver volume. $P$ value was calculated with Student's $t$ test and nonparametric Wilcoxon test. PVE: Portal vein embolization; TACE: transarterial chemoembolization; LV: non-tumorous remnant liver volume; HR: hepatic resection; NA: not available.

alone, but this was not statistically significant. Morbidity was similar in the two groups. The interval of PVE and hepatectomy was comparable, except for one paper showing a shorter period in the PVE-only group ${ }^{[30]}$.

Sequential TACE and PVE are usually performed with TACE first and then PVE after an interval of 2-3 weeks ${ }^{[26-30]}$. By contrast, we have been recommending an inverted order of PVE first and then TACE afterward $^{[24,25]}$ for two reasons: (1) the degree of FLR hypertrophy depends on the interval between PVE and hepatectomy; and (2) PVE is necessary to achieve complete obliteration of portal vein, so PVE after TACE can perform liver infarction.

Moreover, excessive TACE followed by PVE could result in liver infarction or abscess. In the PVE-first approach, however, TACE can be performed delicately with minimal arterial obstruction of the surrounding liver tissue that has undergone PVE.

\section{ADDITIONAL HEPATIC VEIN EMBOLIZATION ON PVE (HPVE)}

Combined preoperative PVE and HVE (known as HPVE) has recently been introduced mainly for colorectal liver metastases and perihilar cancer ${ }^{[32-34]}$. One systematic review ${ }^{[32]}$ containing 68 patients undergoing HPVE has been published. HVE was performed via the transjugular approach, except in one case that used the transhepatic approach. In total, 43 (63.3\%) patients were treated with sequential HVE 1-4 weeks after PVE due to an insufficient increase of FLR, whereas 25 (36.7\%) patients were treated with simultaneous PVE and HVE. The median interval between HPVE and hepatectomy was 21-49 days in simultaneous patients with HPVE. Sequential HPVE allows for delayed liver resection because two procedures are performed in the patient. HPVE was performed successfully in all patients with no mortality and morbidity. The hypertrophy rates of the FLR after HPVE ranged from 33\% to 63.3\%. Liver functional volume assessed via ${ }^{99 m}$ Tc-mebrofenin SPECT/CT following simultaneous HPVE demonstrated a high increase of $64 \%$ (28.1\%-107.5\%) in the FLR function. Major liver resections after preoperative HPVE were performed in $85.3 \%$ of patients. The postoperative morbidity and mortality rates were $10.3 \%$ and $5.1 \%$, respectively. Unfortunately, in this review, patients' HCC and cirrhosis were rarely included, only comprising 5/68 (7.4\%) and 4/68 (6\%) of patients, respectively. 
A retrospective comparative study of HPVE and PVE has been published, which included 31 patients who underwent HPVE and 41 who underwent PVE alone ${ }^{[33]}$. HVE was conducted immediately after the completion of PVE. HVE was performed using the vascular plug, which was $80 \%-100 \%$ wider than the hepatic vein. CT volumetry was performed before and three weeks after HPVE. The mean percentage of FLR hypertrophy from baseline (\%FLR hypertrophy) in the HPVE group was significantly higher than that in the PVE group $(51.2 \%$ vs. $31.9 \%, P=0.018)$. On multivariate analysis, HPVE was found to be one of the independent factors to obtain greater FLR. The kinetic growth rate was defined as the mean degree of hypertrophy divided by the number of weeks. The mean kinetic growth rates were $19 \%$ and $8 \%$ per week in the HPVE and PVE groups, respectively $(P=0.026)$. Intra- and post-operative outcomes were comparable in the HPVE and PVE groups, and there were no specific complications related to the HVE procedure. However, it is important to note that HPVE could pose a higher high risk for intraoperative bleeding and postoperative morbidity after major hepatectomy compared to PVE alone because of the increased number of vascular collaterals between the right and left liver after HVE.

A newer study consisting of 21 patients treated with HPVE and 39 treated with PVE alone ${ }^{[34]}$ demonstrated that the median FLR hypertrophy rate was larger in the HPVE group (135\%, interquartile range: 123\%$154 \%$ ) than in the PVE group (124\%, interquartile range: 107\%-140\%). The degree of hypertrophy did not differ according to tumor type. The median kinetic growth rate was larger in the HPVE group as well (2.9\%/week, interquartile range: $1.9 \%-4.3 \%$ vs. $1.4 \%$ week, interquartile range: $0.7 \%-2.1 \% ; P<0.001)$. Dropout rates were marginally significantly lower in the HPVE group compared with the PVE-only group (5\% vs. $23 \%, P=0.053)$.

More recently, an international multi-center study (DRAGON trial) ${ }^{[35]}$ was published comparing 39 patients undergoing simultaneous HPVE and 160 PVE alone. This study included $18 \%$ and $30 \%$ of patients undergoing TSH in the HPVE and PVE groups, respectively. The HPVE group had greater hypertrophy $(59 \%$ vs. $48 \%, P=0.020)$ and resectability $(90 \%$ vs. $68 \%, P=0.007)$ than the PVE group. Major complications ( $26 \%$ vs. $34 \%, P=0.550)$ and 90 -day mortality $(3 \%$ vs. $16 \%, P=0.065)$ were equivalent.

Nowadays, simultaneous rather than sequential HPVE is recommended for patients with a high risk of PVE failure (i.e., patients with a small FLR volume and underlying injured liver) ${ }^{[32]}$. However, HPVE on patients with cirrhosis should still be applied carefully because HPVE can cause portal hypertension-related complications, such as ascites or variceal bleeding, and carries a risk of portal vein thrombosis. Sequential HPVE is recommended only for patients needing "rescue" (i.e., those with insufficient FLR even after a complete PVE). HPVE is an excellent interventional method to obtain a larger FLR compared with PVE alone. HPVE can be performed via a percutaneous approach under local anesthesia, so it is thought to be less invasive than surgical approaches, including ALPPS. Prospective comparative studies are needed to validate this claim.

\section{RADIATION LOBECTOMY}

RL with glass microspheres loaded with Yttrium-90 (TheraSphere, Nordion, Canada) was first introduced in 2013 with the goal of increasing the FLR rates and potentially controlling the liver tumors in the treated lobe ${ }^{[36]}$. In total, 83 patients (HCC; $n=67$ ) with right unilobar liver tumors were evaluated. The FLR hypertrophy was assessed before and after Y90 radioembolization via dynamic CT/MRI. FLR hypertrophy was observed one month after radioembolization $(P<0.001)$, and this was consistent at all follow-up time points. The median \%FLR hypertrophy reached $45 \%(5 \%-186 \%)$ after nine months $(P<0.001)$. Among 67 patients with HCC, three underwent successful right lobectomy, while six underwent liver transplantation. There was a significant reduction in median tumor volume from 134 to $99 \mathrm{~mL}$ at three months $(P=0.02)$ 
and $56 \mathrm{~mL}$ after nine months. The authors concluded that unilobar Yttrium-90 RE is a beneficial alternative procedure to PVE before major liver resection.

On the other hand, RL for liver metastases showed contrary results. Patients with right-side liver metastases only were treated with right-lobar PVE $(n=141)$ or right-lobar RL $(n=35)^{[37]}$. In total, 26 matched pairs of background factors were identified. FLR volume significantly increased from baseline in patients undergoing PVE compared to RL $(61.5 \%$ vs. $29 \%, P<0.001)$. The follow-up period was longer for patients with RL as well [median, 33 (24-56) days vs. 46 (27-79) days]. In this study, more patients were ineligible for curative hepatectomy in the RL group. However, the authors concluded that RL is one of the suitable modalities for the selected patients because RL can cause contralateral hypertrophy and can minimize the risk of tumor progression in the embolized lobe.

A comparison between short- and long-term outcomes after preoperative PVE and Yttrium-90 RL was conducted in 95 patients with HCC with chronic liver disease ${ }^{[38]}$. There were 73 patients who underwent PVE and 22 patients who underwent RL. In summary, 47\% of patients before PVE required additional procedures for tumor control and $27 \%$ of patients after RL required additional procedures to induce further hypertrophy. Both therapies achieved the goal of FLRs $>40 \%$, but the degree of hypertrophy was significantly higher for patients with RL $(68 \%$ vs. 36\%, $P<0.01)$. Tumor response was significantly better with RL, which achieved a complete response in $50 \%$ of patients. Meanwhile, the resectability rate was higher after PVE ( $85 \%$ for portal vein embolization and $64 \%$ for RL, $P=0.03$ ). Tumor progression was the most common reason precluding surgery. Surgery was not pursed in $18 \%$ of patients after RL because complete tumor control was already achieved. After an intention-to-treat analysis, in patients with initially resectable HCC, five-year survival was significantly better after PVE than after RL ( $52 \%$ vs. $71 \%, P<0.01$ ).

RL is not able to achieve all therapeutic goals, but it can increase liver resectability by inducing hypertrophy of FLRs even for patients with HCC and chronic liver disease. Furthermore, RL monotherapy might provide a better response compared to PVE.

\section{SURGICAL PROCEDURES}

For patients with bilateral unresectable HCCs, one-stage hepatectomy following PVE, TSH, and ALPPS are available options ${ }^{[39-43]}$. The first step of TSH includes tumor enucleation of the FLR, followed by PVE or PVL, and the second step involves major hepatectomy. ALPPS is a novel operative procedure consisting of two steps: PVE or PVL and liver transection with or without tumor enucleation from the FLR, followed by major hepatectomy.

\section{ALPPS vs. PVE focusing on to HCC}

In a systematic review for $\mathrm{HCC}^{[4]}$, the increment in increase of FLR volume was smaller in patients with cirrhosis compared to patients without cirrhosis treated with PVE (24.4\%-38.4\% vs. 39.4\%-49.6\%). In patients with cirrhosis undergoing ALPPS, the increment in increase of FLR volume was relatively larger (38.1\%-71.1\%) and the waiting time for hepatectomy was relatively shorter (6-14 days). ALPPS can clearly result in the shortest waiting time in comparison with not only PVE but also other interventional and surgical procedures ${ }^{[22-32,36,41]}$. PVE can provide relatively good liver regeneration even for patients with HCC concomitant with cirrhosis. Thus, cirrhotic patients are not contraindicated for PVE.

When ALPPS was first started, it was known to have major disadvantages such as high morbidity and mortality ${ }^{[63]}$. Recently, however, the improvement in the selection of patients and the appearance of less invasive modifications from the original technique has improved the results ${ }^{[6,65]}$. The complete and partial 
split in ALPPS were compared in patients with HCC, and the complete split was found to significantly induce a larger growth in FLR volume vs. partial split in chronic hepatitis from the viewpoint of gain in FLR ratio and daily hypertrophy rate ${ }^{[6]]}$. Even though the complete split induced FLR hypertrophy more rapidly than partial split in cirrhosis (hypertrophy rate $32.2 \mathrm{~mL}$ /day vs. $16.9 \mathrm{~mL} /$ day), the difference was less for patients with cirrhosis (\%FLR increment: $14.8 \%$ vs. 11.0\%) than for patients with chronic hepatitis (\%FLR increment: $18.1 \%$ vs. $11.3 \%$ ). Two novel concepts have been developed with respect to classic ALPPS: (1) partial division of the liver parenchyma (partial ALPPS and mini ALPPS); and (2) no division of the liver parenchyma (ALPPS-Tourniquet, radiofrequency ablation, and microwave ablation) ${ }^{[67-71]}$.

Recently, the clinical benefits were debated for ALPPS and conventional hepatectomy after PVE in hepatitis-related HCC patents ${ }^{[42]}$. A total of 148 patients with HCC (hepatitis B: 92.0\%; liver cirrhosis: 56.5\%) underwent FLR modulation (46 ALPPS and 102 PVE). The completion rate of hepatectomy in patients with ALPPS was significantly higher compared to that of patients with PVE ( $97.8 \%$ vs. $67.7 \%, P<0.001)$. Patients undergoing ALPPS had greater increment in FLR/estimated standard liver volume (12.3\% vs. 9.2\%, $P=$ $0.02)$, greater absolute FLR volume increment $(48.8 \% v s .37 .9 \%, P=0.03)$, and shorter time to hepatectomy ( 6 days $v s .48$ days, $P<0.001$ ). \%FLR hypertrophy was larger in chronic hepatitis than in liver cirrhosis $(52.7 \%$ vs. $32.5 \%, P=0.025)$. There was no difference in morbidity $(20.7 \% v s .30 .4 \%, P=0.159)$ and mortality $(6.5 \%$ vs. $5.8 \%, P=1.000)$ between patients who underwent ALPPS and those who underwent hepatectomy following PVE. The five-year OS for patients with ALPPS and PVE was not significantly different as well ( $46.8 \%$ vs. $64.1 \%, P=0.234$ ). The authors concluded that ALPPS was recommended as a salvage option in selected patients with HCC and chronic liver disease.

\section{Functional volumetry for various liver tumors, ALPPS vs. PVE}

FLR volume and function were compared in patients with liver tumors, including HCC after PVE and $\mathrm{ALPPS}^{[43]}$. In total, 72 patients were included: 51 underwent PVE, 12 underwent complete ALPPS, and 9 underwent partial ALPPS (including seven with the laparoscopic approach). Multiphase contrast-enhanced $\mathrm{CT}$ and ${ }^{99 \mathrm{~m}} \mathrm{Tc}$-mebrofenin hepatobiliary scintigraphy were used to assess FLR volume and function, respectively. The median increase in FLR function was 1.8, 2.5, and 1.7 times greater than the median increase in FLR volume for PVE, patients with first complete ALPPS, and partial ALPPS, respectively $(P<$ $0.001, P=0.007$, and $P=0.441)$. The kinetic growth rates for FLR function were significantly higher in the patients with both complete ALPPS $(16.7 \%, P=0.002)$ and partial ALPPS $(9.3 \%, P=0.03)$ compared to the PVE group (4.9\%). The time to achieve a sufficient hypertrophy response was shorter in both ALPPS groups ( 8 and 10 days) than the PVE group (23 days). Among patients undergoing hepatectomy, severe postoperative complication rates $(18 \%, 30 \%$, and $17 \%)$ and 90 -day mortality rates $(2 \%, 25 \%$, and $0 \%)$ were comparable. A laparoscopic partial ALPPS is preferred based on lower morbidity and mortality rates and equivalent functional and volumetric hypertrophy ability.

\section{ALPPS vs. TSH for various liver tumors}

A systematic review was conducted comparing the current status of ALPPS $v s$. TSH for various liver tumors, partially including $\mathrm{HCC}^{[39]}$. TSH is not a common procedure for HCC unlike for colorectal liver metastases. By contrast, ALPPS can provide a more marked hypertrophy rate (50\%-80\% vs. 10\%-40\%), a shorter interval between first and second hepatectomy (7-11 days vs. 20-103 days), and a higher resection rate (80\%-100\% vs. 60\%-90\%) vs. TSH. Compared to patients with normal liver, patients with HCC and chronic liver disease had lower increases in FLR per day (5\%-19\% vs. 9\%-35\%) and less extensive hypertrophy increase in FLR volume ( $40 \%-47 \%$ vs. $76 \%-138 \%$ ). For patients with portal invasion to the right portal vein, PVE is neither technically feasible nor effective, but PVL and ALPPS can help in inducing hypertrophy in HCC cases with portal tumor thrombus. Successful cases have been reported, indicating technical feasibility ${ }^{[72,73]}$. 


\section{ALPPS for HCC vs. colorectal liver metastasis}

The short-term outcome of ALPPS was assessed according to the types of liver tumors [ 35 patients with HCC vs. 225 patients with colorectal liver metastasis (CRLM)]. All patients were registered in the international ALPPS Registry (www.alpps.org) from 2010 to $2015^{[74]}$. In this study, hypertrophy was also rapid and extensive for patients with HCC, but the rates were lower than those for the patients with CRLM ( $47 \%$ vs. $76 \%, P<0.002$ ). Hypertrophy had a negative correlation with the degree of liver fibrosis. The 90day mortality of ALPPS used to treat HCC was almost five times higher than that of CRLM ( $31 \%$ vs. $7 \%, P<$ 0.001). Multivariate analysis demonstrated that patients older than 60 years had a significantly worse overall survival $(P<0.004)$. The authors declared that ALPPS should be performed only in a thoroughly screened patient with HCC population younger than 60 years with low-grade fibrosis.

\section{CONCLUSIONS}

Assessment of FLR function is recently being conducted via ${ }^{99 m}$ Tc-GSA scintigraphy SPECT, ${ }^{99 m}$ Tcmebrofenin hepatobiliary scintigraphy, and Gd-EOB-DTPA MRI. Functional assessment after FLR modification can provide an extended indication for major hepatectomy without increased morbidity and mortality ${ }^{[54-56]}$. PVE can be considered first-line therapy, and additional TACE or HVE is recommended for patients with insufficient FLR after PVE. Surgical approaches are highly invasive procedures; therefore, they should be strictly applied mainly for selected patients with HCC in whom the interventional approach shows insufficient results ${ }^{[42]}$.

\section{DECLARATIONS}

\section{Authors' contributions}

Manuscript writing: Beppu T, Yamamura K

Substantial contributions to conception: Okabe H, Miyata T, Kitano Y, Imai K, Hayashi H, Akahoshi S

Technical supports and interpretation: Beppu T, Yuki H, Yamamura K, Okabe H

\section{Availability of data and materials}

Not applicable.

\section{Financial support and sponsorship}

None.

\section{Conflicts of interest}

All authors declared that there are no conflicts of interest.

\section{Ethical approval and consent to participate}

Not applicable.

\section{Consent for publication}

Not applicable.

\section{Copyright}

(c) The Author(s) 2021.

\section{REFERENCES}

1. Forner A, Reig M, Bruix J. Hepatocellular carcinoma. Lancet 2018;391:1301-14. DOI PubMed

2. Kudo M, Okanoue T; Japan Society of Hepatology. Management of hepatocellular carcinoma in Japan: consensus-based clinical practice manual proposed by the Japan society of hepatology. Oncology 2007;72 Suppl 1:2-15. DOI PubMed

3. Kubota K, Makuuchi M, Kusaka K, et al. Measurement of liver volume and hepatic functional reserve as a guide to decision-making in resectional surgery for hepatic tumors. Hepatology 1997;26:1176-81. DOI PubMed 
4. Schindl MJ, Redhead DN, Fearon KC, Garden OJ, Wigmore SJ; Edinburgh Liver Surgery and Transplantation Experimental Research Group (eLISTER). The value of residual liver volume as a predictor of hepatic dysfunction and infection after major liver resection. Gut 2005;54:289-96. DOI PubMed PMC

5. Yamanaka J, Saito S, Fujimoto J. Impact of preoperative planning using virtual segmental volumetry on liver resection for hepatocellular carcinoma. World J Surg 2007;31:1249-55. DOI PubMed

6. Okabe H, Beppu T, Chikamoto A, et al. Remnant liver volume-based predictors of postoperative liver dysfunction after hepatectomy: analysis of 625 consecutive patients from a single institution. Int J Clin Oncol 2014;19:614-21. DOI PubMed

7. Makuuchi M, Kosuge T, Takayama T, et al. Surgery for small liver cancers. Semin Surg Oncol 1993;9:298-304. DOI PubMed

8. Cucchetti A, Cescon M, Golfieri R, et al. Hepatic venous pressure gradient in the preoperative assessment of patients with resectable hepatocellular carcinoma. J Hepatol 2016;64:79-86. DOI PubMed

9. Molina V, Sampson-Dávila J, Ferrer J, et al. Benefits of laparoscopic liver resection in patients with hepatocellular carcinoma and portal hypertension: a case-matched study. Surg Endosc 2018;32:2345-54. DOI PubMed

10. Rajakannu M, Cherqui D, Ciacio O, et al. Liver stiffness measurement by transient elastography predicts late posthepatectomy outcomes in patients undergoing resection for hepatocellular carcinoma. Surgery 2017;162:766-74. DOI PubMed

11. Mitsumori A, Nagaya I, Kimoto S, et al. Preoperative evaluation of hepatic functional reserve following hepatectomy by technetium99m galactosyl human serum albumin liver scintigraphy and computed tomography. Eur J Nucl Med 1998;25:1377-82. DOI PubMed

12. Kwon AH, Matsui Y, Kaibori M, Ha-Kawa SK. Preoperative regional maximal removal rate of technetium-99m-galactosyl human serum albumin (GSA-Rmax) is useful for judging the safety of hepatic resection. Surgery 2006;140:379-86. DOI PubMed

13. Ziessman HA. Hepatobiliary scintigraphy in 2014. J Nucl Med 2014;55:967-75. DOI PubMed

14. Geisel D, Lüdemann L, Fröling V, et al. Imaging-based evaluation of liver function: comparison of ${ }^{99 \mathrm{~m}} \mathrm{Tc}-\mathrm{meb}$ rofenin hepatobiliary scintigraphy and Gd-EOB-DTPA-enhanced MRI. Eur Radiol 2015;25:1384-91. DOI PubMed

15. Geisel D, Raabe P, Lüdemann L, et al. Gd-EOB-DTPA-enhanced MRI for monitoring future liver remnant function after portal vein embolization and extended hemihepatectomy: A prospective trial. Eur Radiol 2017;27:3080-7. DOI PubMed

16. Lee KC, Kinoshita H, Hirohashi K, Kubo S, Iwasa R. Extension of surgical indications for hepatocellular carcinoma by portal vein embolization. World J Surg 1993;17:109-15. DOI PubMed

17. Imamura H, Shimada R, Kubota M, et al. Preoperative portal vein embolization: an audit of 84 patients. Hepatology 1999;29:1099105. DOI PubMed

18. Azoulay D, Castaing D, Krissat J, et al. Percutaneous portal vein embolization increases the feasibility and safety of major liver resection for hepatocellular carcinoma in injured liver. Ann Surg 2000;232:665-72. DOI PubMed PMC

19. Wei AC, Tung-Ping Poon R, Fan ST, Wong J. Risk factors for perioperative morbidity and mortality after extended hepatectomy for hepatocellular carcinoma. Br J Surg 2003;90:33-41. DOI PubMed

20. Palavecino M, Chun YS, Madoff DC, et al. Major hepatic resection for hepatocellular carcinoma with or without portal vein embolization: perioperative outcome and survival. Surgery 2009;145:399-405. DOI PubMed

21. Siriwardana RC, Lo CM, Chan SC, Fan ST. Role of portal vein embolization in hepatocellular carcinoma management and its effect on recurrence: a case-control study. World J Surg 2012;36:1640-6. DOI PubMed PMC

22. Beppu T, Okabe H, Okuda K, et al. Portal vein embolization followed by right-side hemihepatectomy for hepatocellular carcinoma patients: a Japanese multi-institutional study. J Am Coll Surg 2016;222:1138-48.e2. DOI PubMed

23. Tanaka H, Hirohashi K, Kubo S, Shuto T, Higaki I, Kinoshita H. Preoperative portal vein embolization improves prognosis after right hepatectomy for hepatocellular carcinoma in patients with impaired hepatic function. Br J Surg 2000;87:879-82. DOI PubMed

24. Okabe H, Beppu T, Ishiko T, et al. Preoperative portal vein embolization (PVE) for patients with hepatocellular carcinoma can improve resectability and may improve disease-free survival. J Surg Oncol 2011;104:641-6. DOI PubMed

25. Beppu T, Yamamura K, Okabe H, Imai K, Hayashi H. Oncological benefits of portal vein embolization for patients with hepatocellular carcinoma. Ann Gastroenterol Surg 2021;5:287-95. DOI PubMed PMC

26. Aoki T, Imamura H, Hasegawa K, et al. Sequential preoperative arterial and portal venous embolizations in patients with hepatocellular carcinoma. Arch Surg 2004;139:766-74. DOI PubMed

27. Ogata S, Belghiti J, Farges O, Varma D, Sibert A, Vilgrain V. Sequential arterial and portal vein embolizations before right hepatectomy in patients with cirrhosis and hepatocellular carcinoma. Br J Surg 2006;93:1091-8. DOI PubMed

28. Yoo H, Kim JH, Ko GY, et al. Sequential transcatheter arterial chemoembolization and portal vein embolization versus portal vein embolization only before major hepatectomy for patients with hepatocellular carcinoma. Ann Surg Oncol 2011;18:1251-7. DOI PubMed

29. Terasawa M, Allard MA, Golse N, et al. Sequential transcatheter arterial chemoembolization and portal vein embolization versus portal vein embolization alone before major hepatectomy for patients with large hepatocellular carcinoma: an intent-to-treat analysis. Surgery 2020;167:425-31. DOI PubMed

30. Park GC, Lee SG, Yoon YI, et al. Sequential transcatheter arterial chemoembolization and portal vein embolization before right hemihepatectomy in patients with hepatocellular carcinoma. Hepatobiliary Pancreat Dis Int 2020;19:244-51. DOI PubMed

31. Tustumi F, Ernani L, Coelho FF, et al. Preoperative strategies to improve resectability for hepatocellular carcinoma: a systematic review and meta-analysis. HPB (Oxford) 2018;20:1109-18. DOI PubMed

32. Esposito F, Lim C, Lahat E, et al. Combined hepatic and portal vein embolization as preparation for major hepatectomy: a systematic review. HPB (Oxford) 2019;21:1099-106. DOI PubMed

33. Le Roy B, Gallon A, Cauchy F, et al. Combined biembolization induces higher hypertrophy than portal vein embolization before major liver resection. HPB (Oxford) 2020;22:298-305. DOI PubMed 
34. Kobayashi K, Yamaguchi T, Denys A, et al. Liver venous deprivation compared to portal vein embolization to induce hypertrophy of the future liver remnant before major hepatectomy: a single center experience. Surgery 2020;167:917-23. DOI PubMed

35. Heil J, Korenblik R, Heid F, et al. Preoperative portal vein or portal and hepatic vein embolization: DRAGON collaborative group analysis. Br J Surg 2021;znaa149. DOI PubMed

36. Vouche M, Lewandowski RJ, Atassi R, et al. Radiation lobectomy: time-dependent analysis of future liver remnant volume in unresectable liver cancer as a bridge to resection. J Hepatol 2013;59:1029-36. DOI PubMed PMC

37. Garlipp B, de Baere T, Damm R, et al. Left-liver hypertrophy after therapeutic right-liver radioembolization is substantial but less than after portal vein embolization. Hepatology 2014;59:1864-73. DOI PubMed

38. Bekki Y, Marti J, Toshima T, et al. A comparative study of portal vein embolization versus radiation lobectomy with Yttrium-90 micropheres in preparation for liver resection for initially unresectable hepatocellular carcinoma. Surgery 2021;169:1044-51. DOI PubMed

39. Au KP, Chan ACY. Current status of associating liver partition with portal vein ligation for staged hepatectomy: comparison with twostage hepatectomy and strategies for better outcomes. World J Gastroenterol 2019;25:6373-85. DOI PubMed PMC

40. Zhou Z, Xu M, Lin N, et al. Associating liver partition and portal vein ligation for staged hepatectomy versus conventional two-stage hepatectomy: a systematic review and meta-analysis. World J Surg Oncol 2017;15:227. DOI PubMed PMC

41. Lopez-Lopez V, Robles-Campos R, Brusadin R, et al. ALPPS for hepatocarcinoma under cirrhosis: a feasible alternative to portal vein embolization. Ann Transl Med 2019;7:691. DOI PubMed PMC

42. Chan A, Zhang WY, Chok K, et al. ALPPS versus Portal vein embolization for hepatitis-related hepatocellular carcinoma: a changing paradigm in modulation of future liver remnant before major hepatectomy. Ann Surg 2021;273:957-65. DOI PubMed

43. Lang H, Baumgart J, Mittler J. Associated liver partition and portal vein ligation for staged hepatectomy (ALPPS) registry: what have we learned? Gut Liver 2020;14:699-706. DOI PubMed PMC

44. Saito S, Yamanaka J, Miura K, et al. A novel 3D hepatectomy simulation based on liver circulation: application to liver resection and transplantation. Hepatology 2005;41:1297-304. DOI PubMed

45. Takamoto T, Hashimoto T, Ogata S, et al. Planning of anatomical liver segmentectomy and subsegmentectomy with 3-dimensional simulation software. Am J Surg 2013;206:530-8. DOI PubMed

46. Ohshima S. Volume analyzer SYNAPSE VINCENT for liver analysis. J Hepatobiliary Pancreat Sci 2014;21:235-8. DOI PubMed

47. Kubo S, Shiomi S, Tanaka H, et al. Evaluation of the effect of portal vein embolization on liver function by (99m)tc-galactosyl human serum albumin scintigraphy. J Surg Res 2002;107:113-8. DOI PubMed

48. Nishiyama Y, Yamamoto Y, Hino I, Satoh K, Wakabayashi H, Ohkawa M. 99mTc galactosyl human serum albumin liver dynamic SPET for pre-operative assessment of hepatectomy in relation to percutaneous transhepatic portal embolization. Nucl Med Commun 2003;24:809-17. DOI PubMed

49. Hirai I, Kimura W, Fuse A, Suto K, Urayama M. Evaluation of preoperative portal embolization for safe hepatectomy, with special reference to assessment of nonembolized lobe function with 99mTc-GSA SPECT scintigraphy. Surgery 2003;133:495-506. DOI PubMed

50. Nanashima A, Tobinaga S, Abo T, et al. Relationship of hepatic functional parameters with changes of functional liver volume using technetium-99m galactosyl serum albumin scintigraphy in patients undergoing preoperative portal vein embolization: a follow-up report. J Surg Res 2010;164:e235-42. DOI PubMed

51. Beppu T, Hayashi H, Okabe H, et al. Liver functional volumetry for portal vein embolization using a newly developed $99 \mathrm{mTc}-$ galactosyl human serum albumin scintigraphy SPECT-computed tomography fusion system. J Gastroenterol 2011;46:938-43. DOI PubMed

52. Dinant S, de Graaf W, Verwer BJ, et al. Risk assessment of posthepatectomy liver failure using hepatobiliary scintigraphy and CT volumetry. J Nucl Med 2007;48:685-92. DOI PubMed

53. Graaf W, van Lienden KP, van Gulik TM, Bennink RJ. (99m)Tc-mebrofenin hepatobiliary scintigraphy with SPECT for the assessment of hepatic function and liver functional volume before partial hepatectomy. J Nucl Med 2010;51:229-36. DOI PubMed

54. Rassam F, Olthof PB, Bennink RJ, van Gulik TM. Current modalities for the assessment of future remnant liver function. Visc Med 2017;33:442-8. DOI PubMed PMC

55. Hayashi H, Beppu T, Okabe H, et al. Functional assessment versus conventional volumetric assessment in the prediction of operative outcomes after major hepatectomy. Surgery 2015;157:20-6. DOI PubMed

56. Nilsson H, Blomqvist L, Douglas L, Nordell A, Jonas E. Assessment of liver function in primary biliary cirrhosis using Gd-EOBDTPA-enhanced liver MRI. HPB (Oxford) 2010;12:567-76. DOI PubMed PMC

57. Kan Z, Madoff DC. Liver anatomy: microcirculation of the liver. Semin Intervent Radiol 2008;25:77-85. DOI PubMed PMC

58. Beppu T, Iwatsuki M, Okabe H, et al. A new approach to percutaneous transhepatic portal embolization using ethanolamine oleate iopamidol. J Gastroenterol 2010;45:211-7. DOI PubMed

59. Sofue K, Arai Y, Shimada K, et al. Right portal vein embolization with absolute ethanol in major hepatic resection for hepatobiliary malignancy. Br J Surg 2014;101:1122-8. DOI PubMed

60. Geisel D, Malinowski M, Powerski MJ, et al. Improved hypertrophy of future remnant liver after portal vein embolization with plugs, coils and particles. Cardiovasc Intervent Radiol 2014;37:1251-8. DOI PubMed

61. van den Esschert JW, de Graaf W, van Lienden KP, et al. Volumetric and functional recovery of the remnant liver after major liver resection with prior portal vein embolization : recovery after PVE and liver resection. J Gastrointest Surg 2009;13:1464-9. DOI PubMed PMC

62. Okabe H, Beppu T, Nakagawa S, et al. Percentage of future liver remnant volume before portal vein embolization influences the 
degree of liver regeneration after hepatectomy. J Gastrointest Surg 2013;17:1447-51. DOI PubMed

63. Schnitzbauer AA, Lang SA, Goessmann H, et al. Right portal vein ligation combined with in situ splitting induces rapid left lateral liver lobe hypertrophy enabling 2-staged extended right hepatic resection in small-for-size settings. Ann Surg 2012;255:405-14. DOI PubMed

64. Linecker M, Stavrou GA, Oldhafer KJ, et al. The ALPPS risk score: avoiding futile use of ALPPS. Ann Surg 2016;264:763-71. DOI PubMed

65. Linecker M, Björnsson B, Stavrou GA, et al. Risk adjustment in ALPPS is associated with a dramatic decrease in early mortality and morbidity. Ann Surg 2017;266:779-86. DOI PubMed

66. Chan ACY, Chok K, Dai JWC, Lo CM. Impact of split completeness on future liver remnant hypertrophy in associating liver partition and portal vein ligation for staged hepatectomy (ALPPS) in hepatocellular carcinoma: complete-ALPPS versus partial-ALPPS. Surgery 2017;161:357-64. DOI PubMed

67. Petrowsky H, Györi G, de Oliveira M, Lesurtel M, Clavien PA. Is partial-ALPPS safer than ALPPS? Ann Surg 2015;261:e90-2. DOI PubMed

68. Santibañes E, Alvarez FA, Ardiles V, Pekolj J, de Santibañes M. Inverting the ALPPS paradigm by minimizing first stage impact: the Mini-ALPPS technique. Langenbecks Arch Surg 2016;401:557-63. DOI PubMed

69. Robles R, Parrilla P, López-Conesa A, et al. Tourniquet modification of the associating liver partition and portal ligation for staged hepatectomy procedure. Br J Surg 2014;101:1129-34; discussion 1134. DOI PubMed

70. Wang Q, Yan J, Feng X, et al. Safety and efficacy of radiofrequency-assisted ALPPS (RALPPS) in patients with cirrhosis-related hepatocellular carcinoma. Int J Hyperthermia 2017;33:846-52. DOI PubMed

71. Chen JX, Ran HQ, Sun CQ. Associating microwave ablation and portal vein ligation for staged hepatectomy for the treatment of huge hepatocellular carcinoma with cirrhosis. Ann Surg Treat Res 2016;90:287-91. DOI PubMed PMC

72. Vennarecci G, Laurenzi A, Santoro R, Colasanti M, Lepiane P, Ettorre GM. The ALPPS procedure: a surgical option for hepatocellular carcinoma with major vascular invasion. World J Surg 2014;38:1498-503. DOI PubMed

73. Koga Y, Beppu T, Imai K, et al. Complete remission of advanced hepatocellular carcinoma following transient chemoembolization and portal vein ligation. Surg Case Rep 2018;4:102. DOI PubMed PMC

74. D'Haese JG, Neumann J, Weniger M, et al. Should ALPPS be used for liver resection in intermediate-stage HCC? Ann Surg Oncol 2016;23:1335-43. DOI PubMed 